\title{
Slip Effects and Entropy Generation on Inclined MHD Flow of Williamson Fluid Through a Permeable Wall with Chemical Reaction via DTM
}

\author{
Tunde Abdulkadir Yusuf ${ }^{1}$, Fazle Mabood ${ }^{2 *}$ \\ ${ }^{1}$ Department of Mathematics, University of Ilorin, Ilorin, 240003, Nigeria \\ ${ }^{2}$ Department of Information Technology, Fanshawe College London, ON, London, N5Y 5R6, Canada
}

Corresponding Author Email: fmabood@fanshawec.ca

https://doi.org/10.18280/mmep.070101

Received: 2 December 2019

Accepted: 14 January 2020

\section{Keywords:}

activation energy, Chemical reaction, $M H D$

Williamson fluid, Bejan number, DTM

\begin{abstract}
In the current article, we examine the slip effects for the inclined MHD Williamson fluid over a permeable wall with a chemical reaction. The second law of thermodynamics was applied to examine the aspects of entropy generation. The governing partial differential equations (PDEs) are reduced to ordinary differential equations (ODEs) via appropriately adjusted transformation. The dimensionless developed boundary layer equations have been solved by differential transform method (DTM) for various values of parameters. The most relevant outcomes of the current analysis are that augmented magnetic strength and Williamson fluid parameter undermine the fluid velocity which established a thicker velocity boundary layer while suction/injection show the opposite trend. Another most important outcome is that an increase in suction/injection decreases the entropy generation while it uplifts with Brinkman number. It is also observed that Bejan number decreases with the chemical reaction parameter.
\end{abstract}

\section{INTRODUCTION}

In the present day, the flow of an electrically conducting fluid in a channel in the presence of a magnetic field has captured the interest of researchers due to practical significance in astrophysics and plasma physics to drug targeting. The mechanism of magnetohydrodynamics is that a magnetic field can induce currents in a moving conducting fluid, which in turn creates opposing forces on the fluid. Quite several research papers addressed the flow, heat and mass transfer of Newtonian/non-Newtonian fluid flow with the transverse magnetic field by considering effects of different parameters such as the thermal radiation, porosity, and slip [14]. Moreover, the application of the aligning magnetic field on fluid flow has also received numerous attentions. For instance, Bagewadi and Bhagya [5] examined the influence of the magnetic field on a steady viscous incompressible fluid flow with infinite electrical conductivity. Sandeep and Sugunamma [6] studied the influence of the aligning magnetic field on the unsteady flow of a dusty fluid through a porous medium in a vertical channel. Recently, Endalew and Nayak [7] considered the effects of an inclined magnetic field and chemical reaction of unsteady flow through a porous medium over align plate with thermal radiation. Yadav and Jaiswal [8] scrutinized the impact of an inclined magnetic field on a rotating fluid in the presence of a porous medium. Salman and Abdulhadi [9] used this concept to investigate the two-dimensional peristaltic couple stress fluid flow over an asymmetrically inclined channel through a porous medium.

A literature survey shows that heat and mass transfer problems with a chemical reaction have received a considerable amount of practical usefulness in food processing, polymer production, geothermal engineering and other fields of chemical sciences. So far, research works by several authors [10-17] involved in the diffusion of molecular species relatively to first and higher-order chemical reactions that have considered under different conditions. On the other side, analysis of characteristics of chemically reacting fluid flow in the presence of species and with activation energy has attracted the interest of researchers and has received a considerable amount of attention during the past few decades. For instance, Ahmad et al. [18] investigated the unsteady twodimensional flow of a viscous fluid with binary chemical reaction and Arrhenius activation energy passing through a channel. Mabood et al. [19] utilized the combined effect of the binary chemical reaction and Arrhenius activation energy on electrically conducting fluid with variable thermal conductivity.

We remark at this juncture that all the above studies involve analyses that are only based on the first law of thermodynamics. It is however well known that the convection mode of heat transfer in channel flow is inherently irreversible [20]. To access the nature of the irreversibility, an analysis based on the second law of thermodynamics is required. Some recent studies involving such analysis include the work of Eegunjobi and Makinde [21] who studied the effect of velocity slip flow of a viscous fluid in a permeable channel with entropy generation. Das and Jana [22] extended this to incorporate the magnetic effect. They constructed the analytic solution for the flow and heat transfer and interpreted the results through graphs. The unsteady flow of this viscous fluid with variable viscosity and convective cooling is further investigated by Chinyoka and Makinde [23]. Other interesting results arising from the second-law analysis are discussed by Adesanya et al. [24], Dalir et al. [25], and Opanugat et al. [26]. In all these studies, the researchers argued that it is essential to 
carry out such entropy production analysis to be able to measure the quantity of energy available for work during the convection in fluid flow with the view to upgrade the performance of the system.

This research is motivated by studies on Williamson fluid discussed by Nadeem et al. [27], Nadeem and Akbar [28], and Nagendra et al. [29], the objective is to study the irreversibility analysis due to Williamson fluid subjected to an inclined magnetic field with slip effect and binary chemical reaction. Thus, the present study extends the work of Das and Jana [22] to the non-Newtonian case. To the best of authors' knowledge, this study has not been considered in the previous works of literature. The mathematical model is presented in section 2, the desired solutions of the governing differential equations are obtained in section 3 using the differential transform method (DTM). The analyses of the results are presented in tables and graphs in sections 4 and 5 while section 6 gives the concluding remarks.

\section{PROBLEM FORMULATION}

In Figure 1, a steady flow of an incompressible Williamson fluid through a permeable channel with distance $2 a$ apart with slip is considered. The fluid injection is taken effect at the lower wall while sucked off at the upper wall. The flow is driven due to pressure gradient, also, the fluid is assumed to be acted upon by an external inclined magnetic field of constant strength $B_{0}$ with an angle $\gamma$ placed across the channel while the effect of the applied voltage is negligible. The governing equations arising from the first law of thermodynamics in the presence of thermal radiation is given by Gbadeyan and Yusuf [2], Chinyoka and Makinde [23].

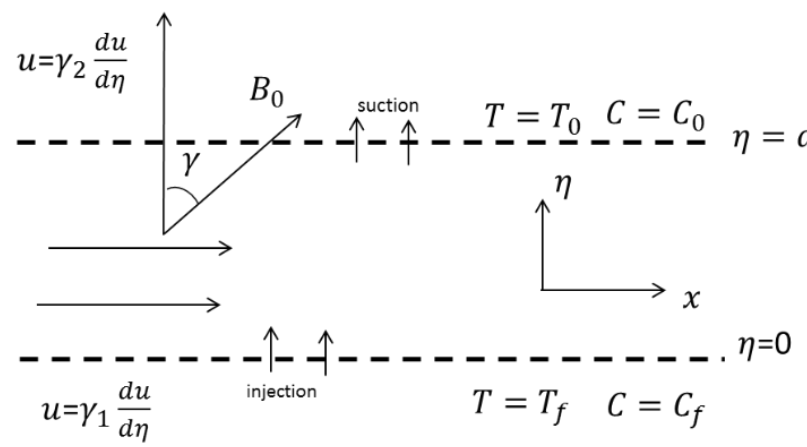

Figure 1. Problem geometry

$$
\begin{gathered}
\rho v_{0} \frac{d u}{d \eta}=-\frac{\partial p}{\partial x}+\mu \frac{d}{d \eta}\left(\frac{d u}{d \eta}+\frac{\Delta}{\sqrt{2}}\left(\frac{d u}{d \eta}\right)^{2}\right)-\frac{\sigma_{o} B_{o}^{2}}{\rho} \sin ^{2} \gamma \\
\rho C_{P} v_{0} \frac{d T}{d \eta}=k \frac{d^{2} T}{d \eta^{2}}+\mu \frac{d u}{d \eta}\left(\frac{d u}{d \eta}+\frac{\Delta}{\sqrt{2}}\left(\frac{d u}{d \eta}\right)^{2}\right) \\
+\sigma_{o} B_{o}^{2} u^{2} \sin ^{2} \gamma-\frac{\partial q_{r}}{\partial \eta} \\
v_{0} \frac{d C}{d \eta}=D_{m} \frac{d^{2} C}{d \eta^{2}}-\delta\left(C-C_{0}\right)\left(\frac{T}{T_{0}}\right) e^{-\frac{E_{s}}{k T}}
\end{gathered}
$$

with the boundary conditions

$$
\begin{aligned}
& u=\gamma_{1} \frac{d u}{d \eta}, T=T_{0}, C=C_{0}, \quad \text { at } \eta=0 \\
& u=\gamma_{2} \frac{d u}{d \eta}, T=T_{f}, C=C_{f} \text { at } \eta=a
\end{aligned}
$$

Expressing $q_{r}$ as a function of temperature the Rosseland approximation is given as [1].

$$
q_{r}=-\frac{4 \sigma_{1}}{3 \chi} \frac{\partial T^{4}}{\partial \eta}=-\frac{16 \sigma_{1}}{3 \chi} T_{0}^{3} \frac{\partial T}{\partial \eta}
$$

Due to poor temperature difference in the fluid flow, the function $T^{m}$ is, therefore, express linearly using the Taylor series expansion with respect to $T_{f}$ and by neglecting the higher-order terms, the approximate term is [18].

$$
\left.\begin{array}{l}
T^{m}=(1-m) T_{f}^{m}+m T_{f}^{m-1} T \\
\left(\frac{T}{T_{f}}\right)^{m} \cong(1-m)+m \frac{T}{T_{f}}
\end{array}\right\}
$$

$u$ is the velocity of the fluid, $a$ is the channel width, $\mu$ is the fluid viscosity, $V_{0}$ is the uniform suction/injection velocity at the channel walls, $k$ is the thermal conductivity coefficient, $\rho$ is the fluid density, $C_{p}$ is the specific heat at constant pressure, $D$ is mass diffusivity, $\delta$ is the chemical reaction parameter, $\beta_{1}$ and $\beta_{2}$ are the slip coefficients, $T$ is the fluid temperature, $T_{f}$ and $T_{0}$ are reference temperature and ambient temperature respectively, $B_{0}$ is the intensity of the magnetic field, $\sigma_{0}$ is the electrical conductivity of fluid, $\sigma_{1}$ is denoted as Stefan Boltzman's constant, while $\chi$ is the mean absorption coefficient, $E$ denotes activation energy parameter, $m$ is a unitless constant exponent fitted rate constants typically lie in the range $-1<w<1$.

Introducing the following non-dimensional quantities

$$
\begin{aligned}
& \eta=\frac{y}{a}, w=\frac{u}{v_{0}}, \alpha=\frac{\sqrt{2 \Delta}}{a}, G=\frac{a^{2}}{\mu v_{0}} \frac{\partial p}{\partial x}, H^{2}=\frac{\sigma_{0} B_{0}^{2} a^{2}}{\mu}, \operatorname{Pe}=\operatorname{Pr} \bullet \mathrm{s} \\
& N=\frac{16 \sigma_{1} T_{0}^{3}}{3 \chi k}, \Omega=\frac{T_{f}-T_{0}}{T_{0}}, K r=\frac{\delta}{a}, B r=\frac{\mu v_{0}^{2}}{k\left(T_{f}-T_{0}\right)}, s=\frac{\rho v_{0} a}{\mu}, \\
& \Pi=\frac{C_{f}-C_{0}}{C_{0}}, \operatorname{Pr}=\frac{C_{p} \mu}{k}, \theta=\frac{T-T_{0}}{T_{f}-T_{0}}, \phi=\frac{C-C_{0}}{C_{f}-C_{0}}, T_{w}=\frac{T_{f}}{T_{0}}, \\
& L=\frac{\mu}{\rho D_{m}}, E_{c}=\frac{v_{0}^{2}}{C_{p}\left(T_{f}-T_{0}\right)}, E=\frac{E_{s}}{k T_{0}}, \xi=\frac{D_{m} C_{0}}{k}, B_{1,2}-\frac{\gamma_{1,2}}{a}, \\
& N_{s}=\frac{E_{G} T_{0}^{2} a^{2}}{k\left(T_{f}-T_{0}\right)^{2}}
\end{aligned}
$$

Given Eqns. (6)-(8), the dimensionless form of the governing equations with their corresponding boundary conditions becomes: 


$$
\begin{gathered}
s \frac{d w}{d y}=G+\frac{d^{2} w}{d y^{2}}\left(1+\alpha \frac{d w}{d y}\right)-H^{2} w \sin ^{2} \gamma=0 \\
P e \frac{d \theta}{d y}=(1+N) \frac{d^{2} \theta}{d y^{2}}+B r\left(\begin{array}{l}
\left.\left(\frac{d w}{d y}\right)^{2}\left(1+\frac{\alpha}{2} \frac{d w}{d y}\right)\right)=0 \\
+H^{2} w^{2} \sin ^{2} \gamma
\end{array}\right)=0 \\
s L \frac{d \phi}{d y}=\frac{d^{2} \phi}{d y^{2}}-\operatorname{LKr} \phi(1+m \Omega \theta) \cdot(1+E+E \Omega \theta)=0
\end{gathered}
$$

And the dimensionless boundary conditions

$$
\begin{aligned}
& w(y)=\mathrm{B}_{1} \frac{d w(y)}{d y}, \theta(y)=\emptyset, \phi=\emptyset \text { at } y=0 \\
& w(y)=\mathrm{B}_{2} \frac{d w(y)}{d y}, \theta(y)=\mathrm{P}, \phi=\mathrm{P} \text { at } y=1
\end{aligned}
$$

In above equations, $S$ is the injection/suction, $\alpha$ is the non-Newtonian parameter, $H$ is the Hartmann number, $G$ is the pressure gradient parameter, $N$ is the radiative flux parameter, $P e$ is the Peclet number, non-dimensional activation energy parameter $E, L$ is the Schmidt number, $B_{1}$ is the lower plate slip parameter, $B_{2}$ is the upper plate slip parameter, $\mathrm{Br}$ is the Brinkman number, $\mathrm{Kr}$ is the chemical reaction rate constant, and $\Omega$ is the temperature difference.

The entropy generation rate $E_{G}$ for the present model is expressed as

$$
\begin{aligned}
& E_{G}=\frac{k}{T_{0}^{2}}\left(1+\frac{16 \sigma_{1}}{3 \chi k} T_{0}^{3}\right)\left(\frac{d T}{d y}\right)^{2}+\frac{\mu}{T_{0}}\left(\frac{d u}{d y}\right)^{2} \\
& \left(1+\frac{\Delta}{\sqrt{2}} \frac{d u}{d \eta}\right)+\frac{\sigma_{0} B_{0}^{2} u^{2}}{T_{0}} \sin ^{2} \gamma+\frac{D_{m}}{C_{0}}\left(\frac{d C}{d y}\right)^{2}
\end{aligned}
$$

The dimensionless entropy generation rate and the irreversibility ratio are

$$
\begin{aligned}
N_{s}= & (1+N)\left(\frac{d \theta}{d y}\right)^{2}+\frac{B r}{\Omega}\left[\left(\frac{d w}{d y}\right)^{2}\left(1+\frac{\alpha}{2} \frac{d w}{d y}\right)+H^{2} w^{2} \sin ^{2} \gamma\right] \\
& +\left(\frac{\Pi}{\Omega}\right)^{2} \xi\left(\frac{d \phi}{d y}\right)^{2}
\end{aligned}
$$

Here, $\Pi$ is the concentration difference, and $\xi$ is the diffusive constant parameter.

Also, defining

$$
\begin{aligned}
& N_{s 1}=(1+N)\left(\frac{d \theta}{d y}\right)^{2} \\
& N_{s 2}=\frac{B r}{\Omega}\left(\frac{d u}{d y}\right)^{2}\left(1+\frac{\alpha}{2} \frac{d u}{d \eta}\right)+\frac{B r}{\Omega} H^{2} w^{2} \sin ^{2} \gamma
\end{aligned}
$$

Therefore, the Bejan number $B e$ and the irreversibility ratio $\Phi$ are defined as

$$
\mathrm{Be}=\frac{N_{s 1}}{N_{s}}=\frac{1}{1+\Phi}, \Phi=\frac{N_{s 2}}{N_{s 1}}
$$

\section{SOLUTION TECHNIQUE AND VALIDATION}

We are interested in obtaining a semi-analytical solution to Eqns. (9)-(13) via the differential transform method (DTM). In this method, the technique of Taylor's analytical solution of the differential equation is used. Transformation rules theorems are applied on the governing differential equation and corresponding the boundary conditions of the system (see Tables 1 and 2) to obtain a set of recurrence relations equations in terms of the differential transforms of the original unknown variables and the expressions of these differential transforms when substituted into the corresponding inverse formulae give the desired finite series solution of the problem. Some of the studies featuring this method was discussed by Opanuga et al. [26], Ayaz [30], Mohammadyari et al. [31].

Consider a function $y(x)$ which is analytic in a domain D and about a point $t=t_{0}$ with $k^{\text {th }}$ derivative. The differential transform of the function is given as:

$$
Y(k)=\frac{1}{k !}\left\{\frac{d^{k} y(x)}{d t^{k}}\right\}_{t=t_{0}}
$$

$y(x)$ is the original function and $Y(k)$ is the transformed function. The inverse transformation is defined as:

$$
y(x)=\sum_{k=0}^{\infty}\left(x-x_{0}\right)^{k} Y(k)
$$

and the finite series is written as

$$
Y(k)=\sum_{k=0}^{j} \frac{\left(x-x_{o}\right)^{k}}{k !}\left\{\frac{d^{k} y(x)}{d t^{k}}\right\}_{t=t_{o} \text { 楼 }}
$$

Some fundamental mathematical operations performed by differential transform method are given in Table 1 below, particularly the ones used in the course of this study.

The iterative schemes are

$$
\begin{aligned}
& W(k+2)=\frac{-1}{(k+2) !}\left\{\begin{array}{l}
G \delta(k)+s(k+1) W(k+1) \\
+\alpha \sum_{r=0}^{k}(r+1) W(r+1) \\
(k-r+2) ! W(k-r+2) \\
-H^{2} W(k) \sin ^{2}(\gamma)
\end{array}\right\} \\
& \theta(k+2)=\frac{-1}{(N+1)(k+2) !}\left\{+B r\left[\begin{array}{l}
-P e(k+1) \theta(k+1) \\
\left.\begin{array}{l}
\sum_{r=0}^{k}(r+1)(k-r+1) \\
W(r+1) W(k-r+1) \\
+\frac{\alpha}{2} \sum_{r=0}^{k} \sum_{t=0}^{r}(t+1) W(t+1) \\
(r+1) W(r+1) \\
(k-r+1) W(k-r+1) \\
+H^{2} \sin ^{2}(\gamma) \sum_{r=0}^{k} W(r) W(k-r)
\end{array}\right]
\end{array}\right\}\right.
\end{aligned}
$$




$$
\phi(k+2)=\frac{-1}{(k+2) !}\left\{\begin{array}{l}
s L(k+1) \phi(k+1)- \\
E \Omega^{2} \sum_{r=0}^{k} \sum_{t=0}^{r} \phi(t) \theta(r) \theta(k-r) \\
-\Omega \sum_{r=0}^{k} \phi(r) \theta(k-r) \\
+(E-1) \phi(k)
\end{array}\right\}
$$

The transformed boundary conditions are

$$
\begin{gathered}
W(0)=B_{1} \cdot A,, W(1)=A, \theta(0)=0, \theta(1)=C, \\
\phi(1)=D,
\end{gathered}
$$

where, $A, C, D$ are constants to be determined. To obtain these constants we employ the second boundary condition, i.e.

$$
\sum_{k=0}^{n} W(k)-B_{2} \sum_{k=0}^{n} k W(k)=0, \sum_{k=0}^{n} \theta(k)=1, \sum_{k=0}^{n} \phi(k)=1
$$

and solving simultaneously with the other governing parameters.

Table 1. Some DTM theorems

\begin{tabular}{|c|c|}
\hline Given Function & Transformed Function \\
\hline$u(x)=f(x) \pm g(x)$ & $W(k)=G(k) \pm H(k)$ \\
\hline$u(x)=\lambda g(x)$ & $W(k)=\lambda G(k)$ \\
\hline$u(x)=f(x) g(x)$ & $W(k)=\sum_{r=0}^{k} F(r) G(k-r)$ \\
\hline$u(x)=\frac{\partial g(x)}{\partial x}$ & $W(k)=(k+1) G(k+1)$ \\
\hline$u(x)=x^{m}$ & $W(k)=\delta(k-m)=\left\{\begin{array}{l}1, k=m \\
0, k \neq m\end{array}\right.$ \\
\hline$u(x)=\frac{\partial^{m} g(x)}{\partial x^{m}}$ & $W(k)=(k+m) ! G(k+m)$ \\
\hline
\end{tabular}

Table 2. DTM theorems used for the boundary conditions

\begin{tabular}{|c|c|c|c|}
\hline $\begin{array}{c}\text { Original } \\
\text { B.C. }\end{array}$ & $\begin{array}{c}\text { Transforme } \\
\text { d B.C. }\end{array}$ & $\begin{array}{c}\text { Original } \\
\text { B.C. }\end{array}$ & Transformed B.C. \\
\hline$f(0)=0$ & $F(0)=0$ & $f(1)=0$ & $\sum_{k=0}^{\infty} F(k)=0$ \\
\hline$\frac{d f(0)}{d \eta}=0$ & $F(1)=0$ & $\frac{d f(1)}{d \eta}$ & $\sum_{k=0}^{\infty} k F(k)=0$ \\
\hline$\frac{d^{2} f(0)}{d \eta^{2}}=0$ & $F(2)=0$ & $\frac{d^{2} f(1)}{d \eta^{2}}$ & $\sum_{k=0}^{\infty} k(k-1) F(k)=0$ \\
\hline$\frac{d^{3} f(0)}{d \eta^{3}}=0$ & $F(3)=0$ & $\frac{d^{3} f(1)}{d \eta^{3}}$ & $\begin{array}{l}\sum_{k}^{\infty} k(k-1)(k-2) \\
k=0 \\
F(k)=0\end{array}$ \\
\hline
\end{tabular}

\section{CODE VALIDATION}

To validate the solution obtained by DTM, the exact solution of the Newtonian flow $\alpha=0$ of the model has been considered earlier by Das and Jana [22] when $\gamma=\frac{\pi}{2}$. The solution of the momentum equation and its corresponding boundary condition is given as:

$$
w(y)=\frac{G}{H^{2}}+\frac{G\left[\begin{array}{l}
e^{m_{1} y}\left\{\left(1-B_{1} m_{2}\right)-\left(e^{m_{2}}-B_{2} m_{2} e^{m_{2}}\right)\right\} \\
+e^{m_{2} y}\left\{\left(1-B_{1} m_{1}\right)-\left(e^{m_{1}}-B_{2} m_{1} e^{m_{1}}\right)\right\}
\end{array}\right]}{H^{2}\left\{\begin{array}{l}
\left(1-B_{1} m_{1}\right)\left(e^{m_{2}}-B_{2} m_{2} e^{m_{2}}\right) \\
-\left(e^{m_{1}}-B_{2} m_{1} e^{m_{1}}\right)\left(1-B_{1} m_{2}\right)
\end{array}\right\}}
$$

where,

$$
m_{1}=\frac{1}{2}\left\{s-\left(s^{2}+4 H^{2}\right)^{\frac{1}{2}}\right\}, m_{2}=\frac{1}{2}\left\{s+\left(s^{2}=4 H^{2}\right)^{\frac{1}{2}}\right\}
$$

A very good agreement is reported in Table 3, Also convergent of the series solution is displayed in Table 4; confident of this solution is therefore reliable.

Table 3. Comparison between the exact and DTM solution of

$$
\begin{aligned}
\text { velocity profile for } \gamma & =\frac{\pi}{2}, H^{2}=1, G=1, s=0.1, \alpha=0, \\
B_{1} & =0.1, B_{2}=0.1
\end{aligned}
$$

\begin{tabular}{|c|c|c|}
\hline$y$ & DTM $w(y)$ & Exact $w(y)$ \\
\hline 0.0 & 0.03619078267 & 0.03619078 \\
\hline 0.1 & 0.06778459159 & 0.06778459 \\
\hline 0.2 & 0.09031919293 & 0.09031919 \\
\hline 0.3 & 0.10393020550 & 0.10393020 \\
\hline 0.4 & 0.10866485260 & 0.10866485 \\
\hline 0.5 & 0.10448154920 & 0.10448154 \\
\hline 0.6 & 0.09124858994 & 0.09124858 \\
\hline 0.7 & 0.06874192040 & 0.06874192 \\
\hline 0.8 & 0.03664195382 & 0.03664195 \\
\hline 0.9 & -0.005547060308 & -0.00554706 \\
\hline 1.0 & -0.05811996948 & -0.05811996 \\
\hline
\end{tabular}

\section{DISCUSSION OF RESULTS}

Comprehensive analysis has shown in Figures 1-8 for the influence of the governing parameters on the dimensionless velocity, temperature, and concentration profiles. Further, the effects of these parameters on skin friction, Nusselt number, and Sherwood number are also shown in Table 4. The influence of magnetic parameter $H^{2}$, non-Newtonian parameter $\alpha$, thermal radiation parameter $N$, chemical reaction rate parameter $K r$ and the suction/injection parameter $S$ on the friction factor, heat transfer rate number and the mass transfer rate is shown in Table 4. It was shown from this table that increase parameters $H^{2}$ causes a reduction in friction factor at both walls. The rate of heat transfer decreases at the wall $y=0$ reduces with in $H^{2}$ whereas it rises at the wall 
$y=1$. At $y=0$, increase in the non-Newtonian parameter lessens the friction factor and the mass transfer rate but enhances the rate of heat transfer and the opposite trend is noticed at the wall $y=1$. The rate of heat transfer is seen to increase at $y=0$ with rising thermal radiation parameter whereas it reduces at the wall $y=1$. An increase in chemical reaction rate suppresses the rate of mass transfer at lower wall $y=0$ but enhances at the upper wall $y=1$. An increase in the suction/injection parameter reduces the friction factor and Sherwood at the lower wall while the reversed trend is noticed at the upper wall, whereas the rate of heat transfer decreases at both walls.

Table 4. Convergence DTM results for $\gamma=\frac{\pi}{2}, H^{2}=1, G=1, s=0.1, \alpha=0, B_{1}=0.1, B_{2}=0.1, E=0.6, \Omega=1, N=1, P e=2, L=3, K r=1$, $y=0.1$

\begin{tabular}{|c|c|c|c|c|c|c|}
\hline$i$ & $w_{i}$ & $\sum_{i=0}^{n} w_{i}$ & $\theta_{i}$ & $\sum_{i=0}^{n} \theta_{i}$ & $\phi_{i}$ & $\sum_{i=0}^{n} \phi_{i}$. \\
\hline 0 & 0.03144176037 & 0.0314418 & 0.000000000 & 0.0000000 & 0.0000000000 & 0.0000000 \\
\hline 1 & 0.3144176037 & 0.0628835 & 0.6234501537 & 0.0623450 & 0.4021218550 & 0.0402121 \\
\hline 2 & -0.3668829472 & 0.0592147 & 0.1840195400 & 0.0641852 & 0.3015913912 & 0.0432280 \\
\hline 3 & 0.02419044977 & 0.0592388 & 0.2509409493 & 0.0644361 & 0.2312200666 & 0.0434593 \\
\hline 4 & -0.0521795958 & 0.0592336 & -0.057380022 & 0.0644304 & 0.07763604832 & 0.0434671 \\
\hline 5 & -0.0084960051 & 0.0592336 & 0.0232647535 & 0.0644306 & 0.00927842076 & 0.0434671 \\
\hline 6 & -0.0044414595 & 0.0592336 & -0.021074840 & 0.0644306 & -0.0106504573 & 0.0434671 \\
\hline 7 & -0.0017073892 & 0.0592336 & -0.003220534 & 0.0644306 & -0.0111973246 & 0.0434671 \\
\hline
\end{tabular}

(a) Velocity profile

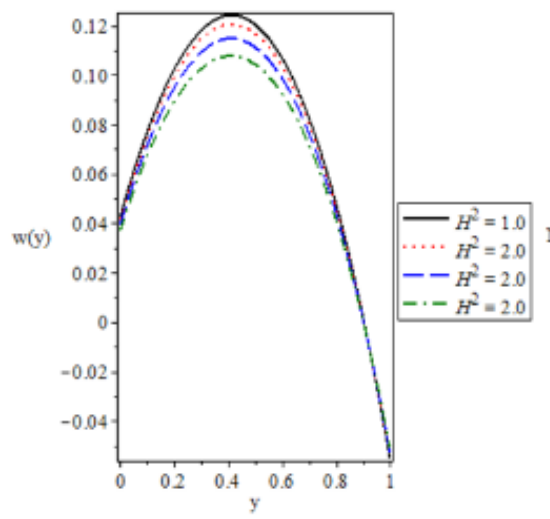

(b) Entropy generation

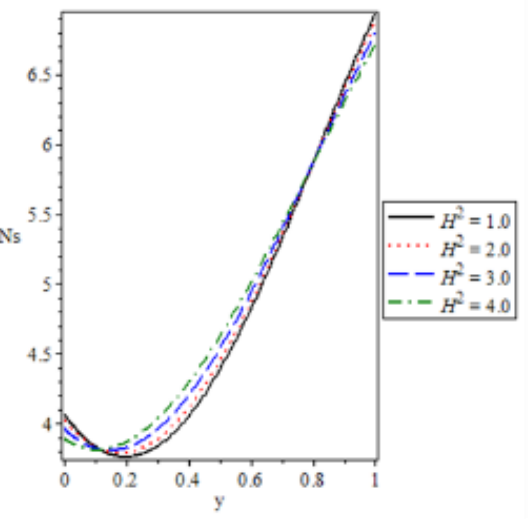

(c) Bejan number

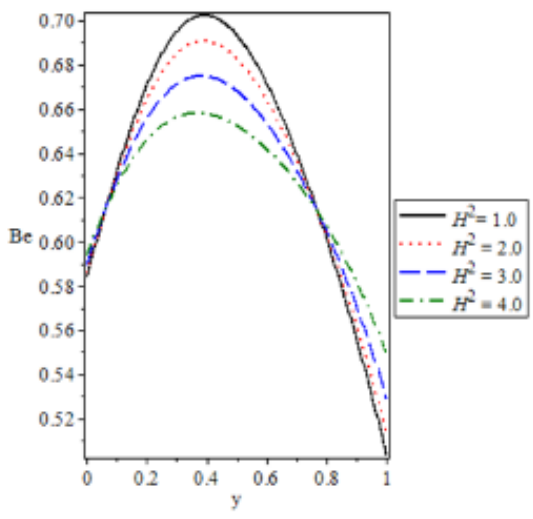

Figure 2. Influence of magnetic field parameter $H^{2}$ for $s=0.5, \alpha=0.2, B_{1}=B_{2}=0.1, G=1, N=2, B r=5, P e=1$,

$$
E=0.6, L=3, m=1, \Omega=1, \Pi=0.6, K r=1, \gamma=\pi / 6, \xi=1
$$

(a) Velocity profile

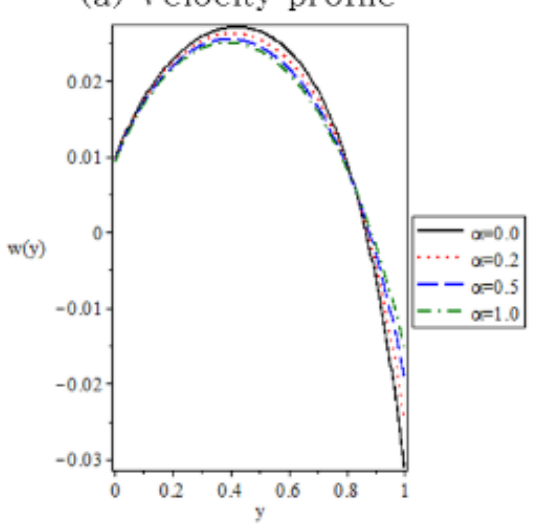

(b) Entropy generation

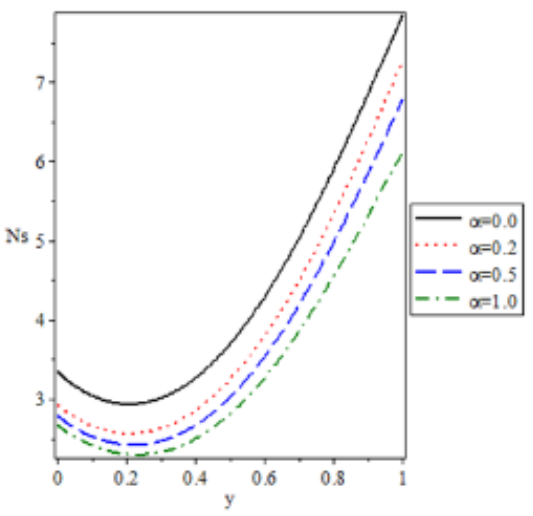

(c) Bejan number

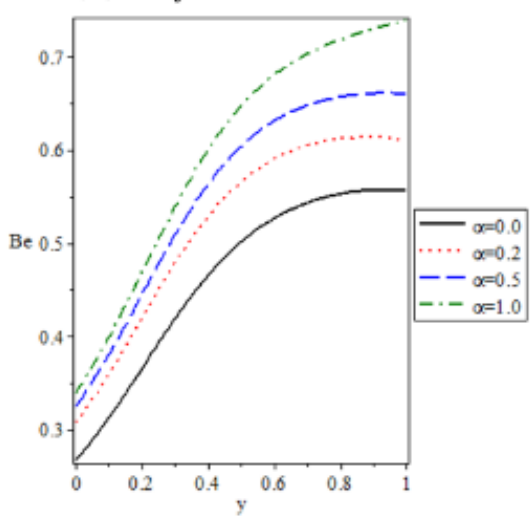

Figure 3. Influence of Williamson parameter $\alpha$ for $s=0.5, \mathrm{H}^{2}=2, B_{1}=B_{2}=0.1, G=1, N=2, B r=5, P e=1$,

$$
E=0.6, L=3, m=1, \Omega=1, \Pi=0.6, K r=1, \gamma=\pi / 6, \xi=1
$$


(a) Velocity profile

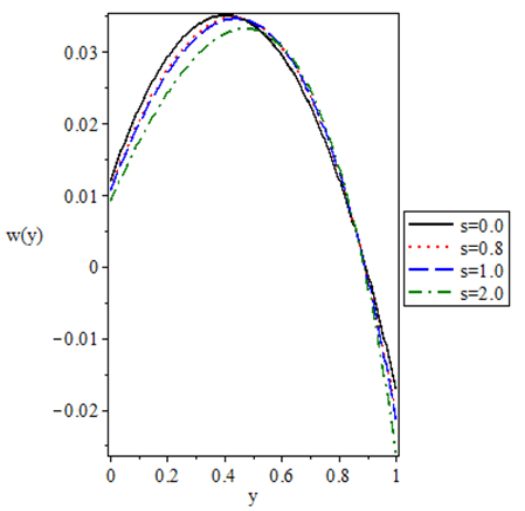

(b) Entropy generation

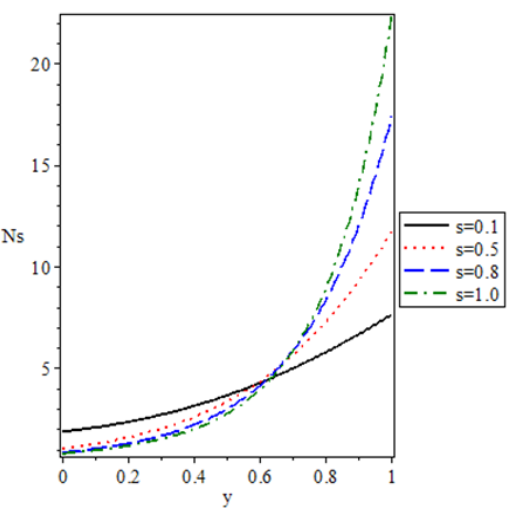

(c) Bejan number

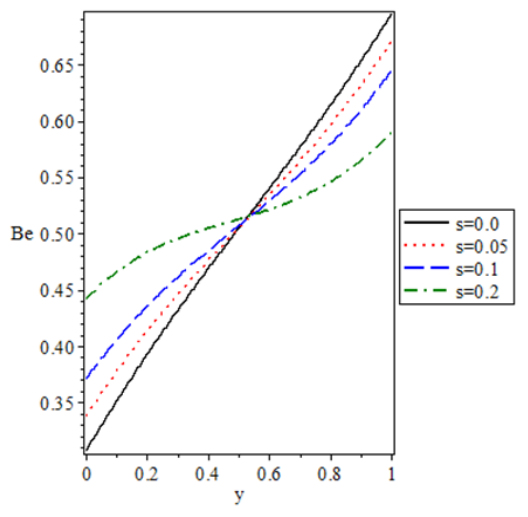

Figure 4. Influence of Suction/injection parameter $S$ for $\alpha=0.2, \mathrm{H}^{2}=2, B_{1}=B_{2}=0.1, G=0.3, N=2, B r=5, P e=2$,

$$
E=0.6, L=3, m=1, \Omega=1, \Pi=0.6, K r=1, \gamma=\pi / 6, \xi=1
$$

(a) Temperature profile

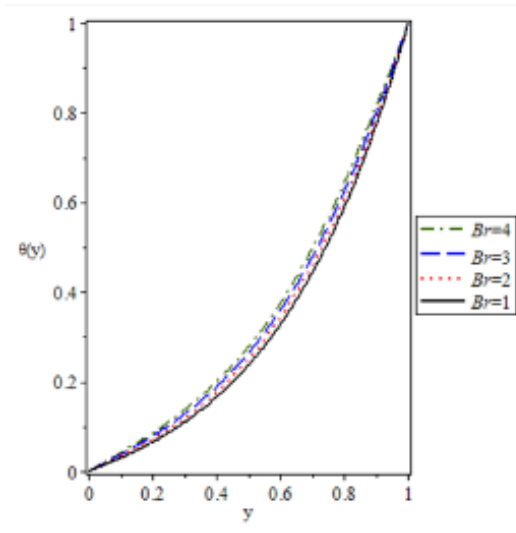

(b) Entropy generation

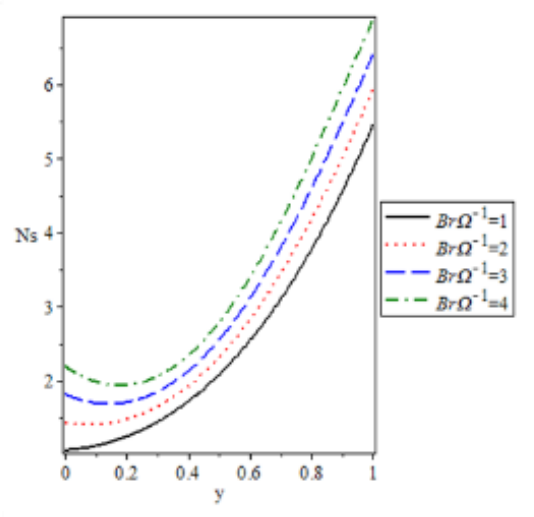

(c) Bejan number

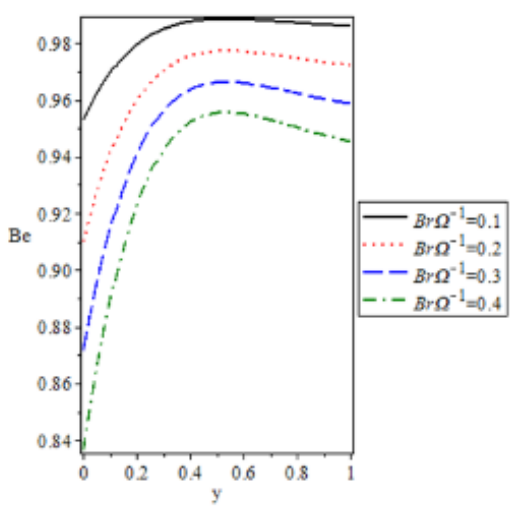

Figure 5. Influence of angle of inclination parameter $B r$ for $s=0.05, \mathrm{H}^{2}=2, B_{1}=B_{2}=0.1, G=0.5, N=1, \gamma=\pi / 6 ? P e=1$,

$$
E=0.6, L=3, m=1, \Omega=1, \Pi=0.6, K r=1, \alpha=0.2, \xi=1
$$

Figure 2 depicts the effect of the magnetic field parameter $H$ on the flow profile. Figure 2(a) shows a consistent depreciation in the velocity profile as the magnetic field increases. This deceleration is due to the Lorentzian magnetic drag opposing the fluid flow. It is shown in Figure 2(b) that the presence of a magnetic field causes more entropy is generated in fluid and decrease is noticed in the entropy profile toward the walls of the plate. The frictional heat and the diffusive irreversibility dominate over the transfer irreversibility as depicted in Figure 2(c).

The response of the varying values of the non-Newtonian Williamson fluid parameter $\alpha$ on the flow profiles is illustrated in Figure 3. Figure 3(a) depicts that the Williamson fluid parameter is seen to decrease both the profiles of the velocity and entropy generation rate. Physically, the Williamson fluid parameter measures the impact of viscosity to elasticity. Due to low resistance to flow, it causing a decrease in the velocity profile. The rate of entropy generation is seen to increase significantly across the channel with increasing numbers of Williamson fluid parameter. Moreover, the heat transfer dominates the irreversibility ratio throughout the channel.

Figure 4 illustrates the influence of the fluid suction/injection parameter on the flow profiles. Due to a continual rise in fluid flow injecting through the lower plate and sucked off through the upper wall, a symmetrical pattern is noticed at the centerline and almost the upper plate (see Figure 4(a)). At the plate with injection In Figure 4(b), the entropy generation rate decreases with the rise in fluid suction/injection parameter while opposite behavior is observed at the plate with suction. With an increase in the fluid suction/injection parameter, the irreversibility ratio contributed equally at the centerline of the channel.

Figure 5 Present the angle of Brinkman number $\mathrm{Br}$ on the flow profiles. As depicted in Figure 5(a), an increase in Brinkman number upsurge the temperature distribution due to an increase in fluid kinetic energy. Consequently, the rate of entropy generation increases with a rise in Brinkman number, while Brinkman number increases the heat transfer dominates over the fluid viscosity as shown in Figures 5(a) and 5(b).

We observed in Figure 6(a) that the increase of the thermal radiation parameter $N$ results in a rise in the temperature distribution throughout the channel. This phenomenon is because the large value of the thermal radiation parameter implies a larger surface heat flux which leads to an increase in the temperature distribution of the fluid. The higher value of the thermal radiation parameter increases the rate of entropy generation as depicted in Figure 6(b). Consequently, it is displayed in Figure 6(c) that the dominance of heat transfer irreversibility over viscous irreversibility increases with the 
thermal radiation parameter.

The effect of the chemical reaction parameter $K r$ on the flow profiles is shown in Figure 7. A higher value of chemical reaction parameter implies lower molecular diffusivity, it is observed in Figure 7(a) that lesser species is diffused thereby causing a reduction in the concentration distribution. Consequently, Figure 7(b) shows that the rate of entropy generation is suppressed at the lower plate while reverse behavior is noticed at the upper plate. However, as depicted in Figure 7(c) that the Bejan number is seen to decrease with an increasing number of chemical reaction parameter. This shows that an increase in this parameter breaks the symmetry due to a continual decrease in the diffusion. Finally, drawn the flow pattern of the current study in Figure 8(a) and (b).

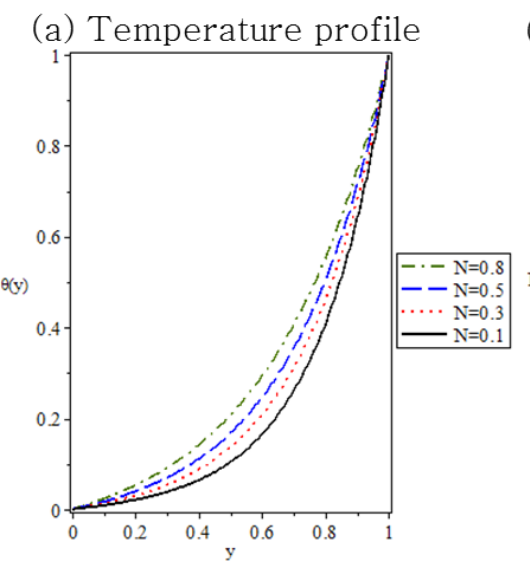

(b) Entropy generation

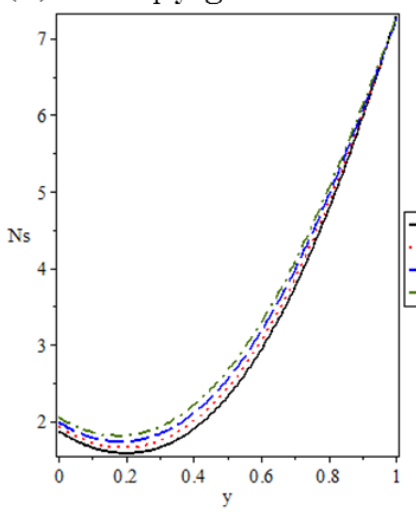

(c) Bejan number
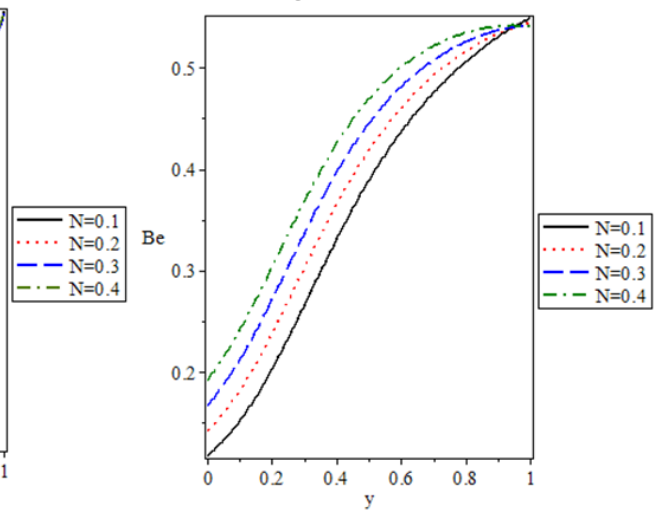

Figure 6. Influence of Thermal radiation parameter $N$ for $s=0.05, \mathrm{H}^{2}=2, B_{1}=B_{2}=0.1, G=1, \alpha=0.2, B r=5$ ?, $P e=1$,

$$
E=0.6, L=3, m=1, \Omega=1, \Pi=0.6, K r=1, \gamma=\pi / 6, \xi=1
$$

(a) Concentration profile

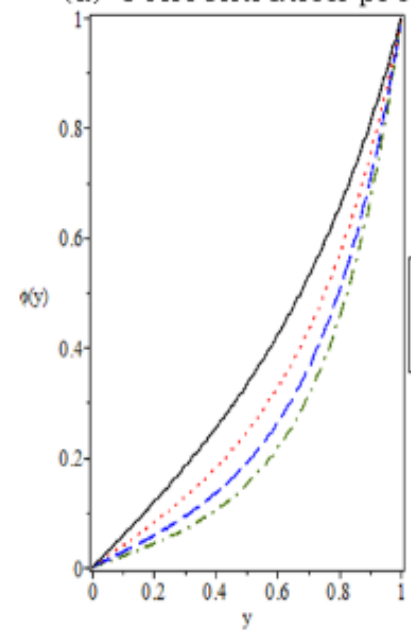

(b) Entropy generation

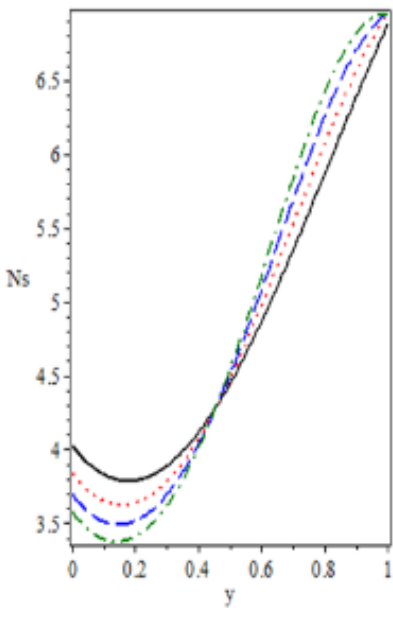

(c) Bejan number

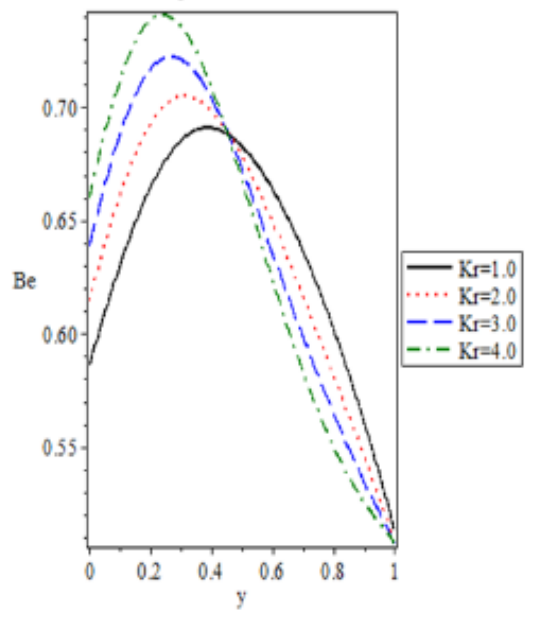

Figure 7. Influence of chemical reaction parameter $K r$ for $s=0.05, \mathrm{H}^{2}=2, B_{1}=B_{2}=0.1, G=1, N=2, B r=5, P e=1$, $E=0.6, L=3, m=1, \Omega=1, \Pi=0.6, \alpha=0.2, \gamma=\pi / 6, \xi=1$
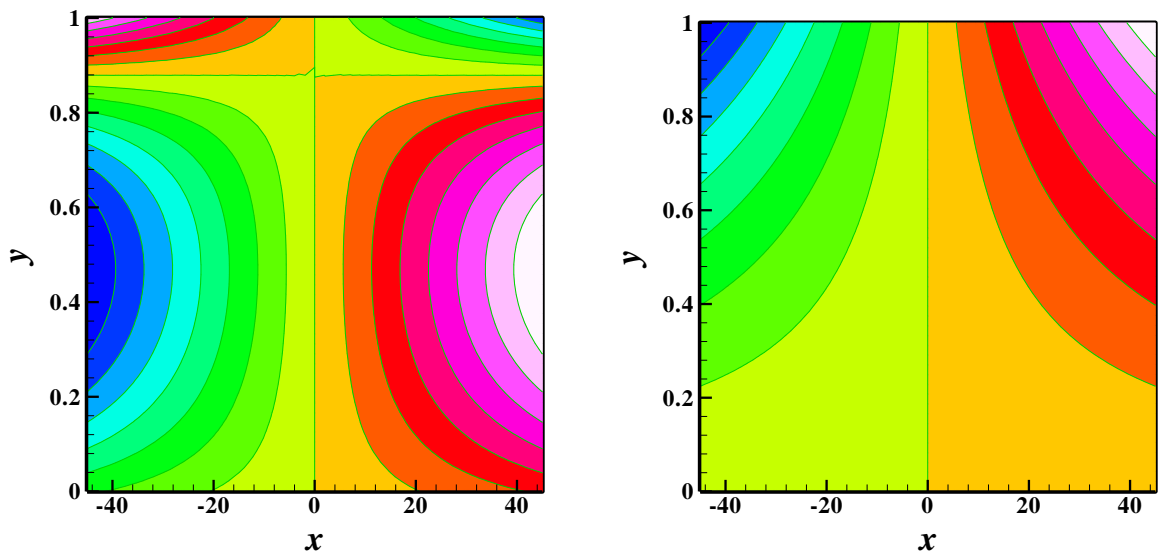

Figure 8. (a) Streamlines (b) Isotherms pattern of the present study 
Table 5. Values of $\mathrm{w}^{\prime}(y), \theta^{\prime}(y), \phi^{\prime}(y)$ when $G=1, \gamma=\frac{\pi}{6}, B_{1}=B_{2}=0.1, B r=2, P e=3, E=0.6, L=2, m=1, \Omega=0.6$

\begin{tabular}{|c|l|c|c|c|c|c|c|c|c|c|}
\hline $\mathrm{H}$ & $\alpha$ & $\mathrm{N}$ & $\mathrm{K}$ & $\mathrm{s}$ & $\mathrm{w}(0)$ & $-\mathrm{w}(1)$ & $\theta(0)$ & $\theta(1)$ & $\phi(0)$ & $\phi(1)$ \\
\hline 1 & 0.2 & 2 & 0.5 & 0.1 & 0.3790692 & 0.6399072 & 0.5987834 & 1.5358174 & 0.7187397 & 1.6396473 \\
\hline 2 & 0.2 & 2 & 0.5 & 0.1 & 0.3553057 & 0.6132564 & 0.5980300 & 1.5392221 & 0.7187492 & 1.6395899 \\
\hline 3 & 0.2 & 2 & 0.5 & 0.1 & 0.3221001 & 0.5768956 & 0.5968921 & 1.5438227 & 0.7187678 & 1.6395044 \\
\hline 3 & 0.2 & 2 & 0.5 & 0.1 & 0.3221001 & 0.5768956 & 0.5968921 & 1.5438227 & 0.7187678 & 1.6395044 \\
\hline 3 & 0.4 & 2 & 0.5 & 0.1 & 0.3221001 & 0.5768956 & 0.5969901 & 1,5428806 & 0.7187649 & 1.6395151 \\
\hline 3 & 0.6 & 2 & 0.5 & 0.1 & 0.3124296 & 0.6730513 & 0.5970966 & 1.5414187 & 0.7187601 & 1.6395329 \\
\hline 3 & 0.2 & 1 & 0.5 & 0.1 & 0.3221001 & 0.5768956 & 0.4512420 & 1.8716379 & 0.7243002 & 1.6272579 \\
\hline 3 & 0.2 & 2 & 0.5 & 0.1 & 0.3221001 & 0.5768956 & 0.5968921 & 1.5438227 & 0.7187678 & 1.6395044 \\
\hline 3 & 0.2 & 3 & 0.5 & 0.1 & 0.3221001 & 0.5768956 & 0.6831496 & 1.3933475 & 0.7158798 & 1.6456134 \\
\hline 3 & 0.2 & 2 & 0.5 & 0.1 & 0.3221001 & 0.5768956 & 0.5968921 & 1.5438227 & 0.7187678 & 1.6395044 \\
\hline 3 & 0.2 & 2 & 0.8 & 0.1 & 0.3221001 & 0.5768956 & 0.5968921 & 1.5438227 & 0.6321980 & 1.9224724 \\
\hline 3 & 0.2 & 2 & 1.0 & 0.1 & 0.3221001 & 0.5768956 & 0.5968921 & 1.5438227 & 0.5821807 & 2.0983371 \\
\hline 3 & 0.2 & 2 & 0.5 & 0.1 & 0.3221001 & 0.5768956 & 0.5968921 & 1.5438227 & 0.7187678 & 1.6395044 \\
\hline 3 & 0.2 & 2 & 0.5 & 0.3 & 0.3155117 & 0.6067958 & 0.5968365 & 1.5417154 & 0.5813439 & 1.8614655 \\
\hline 3 & 0.2 & 2 & 0.5 & 0.5 & 0.3087681 & 0.6384658 & 0.5967627 & 1.5394734 & 0.4645763 & 2.1048017 \\
\hline
\end{tabular}

\section{CONCLUSIONS}

A semi-analytical method has been employed to investigate the slip effects on the flow and heat transfer mechanisms of Williamson fluids through a porous channel. In the present study, the effect of a binary chemical reaction and Arrhenius activation energy is taken into account to stimulate the mass transfer. The result for a limiting case of this present study when $\beta=0$, was further compared with that obtained by Das and Jana [23]. Some of the findings from the current study are:

- The fluid velocity is an increasing function of Williamson fluid and magnetic parameters

- Thermal boundary layer thickens due to radiation parameter

- Concentration decreases with an increase in chemical reaction parameter

- An increase in Brinkman number causes the increase of entropy generation

- Bejan number is decreasing function of chemical reaction parameter

- The skin friction coefficient suppresses with magnetic and non- Newtonian parameters.

- The Nusselt number increases with thermal radiation parameters at both walls, whereas the Sherwood reduces with chemical reaction rate parameter at $y=0$ and strengthen at $y=1$

\section{REFERENCES}

[1] Megahed, A.M. (2019). Williamson fluid flow due to a nonlinearly stretching sheet with viscous dissipation and thermal radiation. Journal of the Egyptian Mathematical Society, 27: 12. http://doi.org/10.1186/s42787-0190016-y

[2] Gbadeyan, J.A., Yusuf, T.A. (2019). Effect of nonlinear partial slip and thermal radiation on Oldroyd 8-constant fluid in a channel with convective boundary condition. Heat Transfer-Asian Res., 49(2): 755-778. https://doi.org/10.1002/htj.21637

[3] Mabood, F., Lorenzini, G., Pochai, N. Shateyi, S. (2018). Homotopy analysis method for radiation and hydrodynamic-thermal slips effects on MHD flow and heat transfer impinging on stretching sheet. Defect and Diffusion $\quad$ Forum, 388: 317-327. https://doi.org/10.4028/www.scientific.net/DDF.388.31 7

[4] Nagendra, N., Amanulla., C.H., Reddy, M.S. (2017). Slip effects on MHD flow of a Williamson fluid from an isothermal sphere: A numerical study. AMSE JOURNALS-AMSE IIETA publication-2017-Series: Modelling B, 86(3): 782-807. https://doi.org/10.18280/mmc_b.860311

[5] Bagewadi, C.S., Bhagya, S. (2011). Solutions in variably inclined MHD flows. Journal of Applied Fluid Mechanics, 4(4): 77-83.

[6] Sandeep, N., Sugunamma, V. (2013). Effect of inclined magnetic field on unsteady free convection flow of a dusty viscous fluid between two infinite flat plates filled by a porous medium. International Journal of Applied Mathematics and Modeling, 1(1): 16-33.

[7] Endalew, M.F., Nayak, A. (2018). Thermal radiation and inclined magnetic field effects on MHD flow past a linearly accelerated inclined plate in a porous medium with variable temperature, Heat Transfer-Asian Res., 48(1): 42-61. https://doi.org/10.1002/htj.21367

[8] Yadav, P. K., Jaiswal, S. (2018). Influence of an inclined magnetic field on the Poiseuille flow of immiscible micropolar-Newtonian fluids in the porous medium. Canadian Journal of Physics, 96(9): 1016-1028. https://doi.org/10.1139/cjp-2017-0998

[9] Salman, M.R., Abdulhadi, A.M. (2018). Influence of heat and mass transfer on inclined (MHD) peristaltic of pseudoplastic nanofluid through the porous medium with couple stress in an inclined asymmetric channel. Journal of Physics: Conference Series, 1032: 012043. https://doi.org/10.1088/1742-6596/1032/1/012043

[10] Takhar, H.S., Chamkha, A.J., Nath, G. (2000). Flow and mass transfer on a stretching sheet with a magnetic field and chemically reactive species. International Journal of Engineering Science, 38(12): 1303-1314. https://doi.org/10.1016/S0020-7225(99)00079-8

[11] Rahman, M., Al-Lawatia, M. (2010). Effects of higher order chemical reaction on micropolar fluid flow on a power law permeable stretched sheet with variable 
concentration in a porous medium. Can. J. Chem. Eng. 88(1): 23-32. https://doi.org/10.1002/cjce.20244

[12] Rout, B.R., Parida, S.K., Panda, S. (2013). MHD heat and mass transfer of chemical reaction fluid flow over a moving vertical plate in presence of heat source with convective surface boundary condition. International Journal of Chemical Engineering, 2013. http://dx.doi.org/10.1155/2013/296834

[13] Srikanth, G.V.P.N., Srinivasa, G., Babu, B.S. (2015). Characterization of chemical reaction on heat transfer through the nano fluid. Procedia Materials Science, 10: 10-18. https://doi.org/10.1016/j.mspro.2015.06.018

[14] Zhu, J., Zheng, L., Zheng, L., Zhang, X. (2015). Secondorder slip MHD flow and heat transfer of nanofluids with thermal radiation and chemical reaction. Applied Mathematics and Mechanics, 36: 1131-1146. https://doi.org/10.1007/s10483-015-1977-6

[15] Mabood, F., Ibrahim, S. M., Lorenzini, G. (2017). Chemical reaction effects on MHD rotating fluid over a vertical plate embedded in porous medium with heat source. Journal of Engineering Thermophysics, 26(3): $399-415$

[16] Mabood, F., Khan, W.A., Ismail, A.I.M. (2015). MHD stagnation point flow and heat transfer impinging on stretching sheet with chemical reaction and transpiration. Chemical Engineering Journal, 273: 430-437. https://doi.org/10.1016/j.cej.2015.03.037

[17] Malik, M.Y., Khalil-Ur-Rehman. (2016). Effects of second order chemical reaction on MHD free convection dissipative fluid flow past an inclined porous surface by way of heat generation: a lie group analysis. Inf. Sci. Lett. 5(2): 35-45.

[18] Ahmad, S., Farooq, M., Mir, N.A., Anjum, A., Javed, M. (2019). Magneto-hydrodynamic flow of squeezed fluid with binary chemical reaction and activation energy. $\mathrm{J}$. Cent. South Univ., 26: 1362-1373. https://doi.org/10.1007/s11771-019-4092-9

[19] Mabood, F., Nayak, M.K., Chamkha, A.J. (2019). Heat transfer on the cross flow of micropolar fluids over a thin needle moving in a parallel stream influenced by binary chemical reaction and Arrhenius activation energy. The European Physical Journal Plus, 134: 427. https://doi.org/10.1140/epjp/i2019-12716-9

[20] Bejan, A. (1996). Entropy Generation Minimization. CRC Press: New York, NY, USA.

[21] Eegunjobi, A.S., A.S., Makinde, O. D. (2012). Combined effect of buoyancy force and navier slip on entropy generation in a vertical porous channel. Entropy, 14(6):
1028-1044. https://doi.org/10.3390/e14061028

[22] Das, S., Jana, R.N. (2014). Entropy generation due to MHD flow in a porous channel with Navier slip. Ain Shams Engineering Journal, 5(2): 575-584. https://doi.org/10.1016/j.asej.2013.11.005

[23] Chinyoka, T., Makinde, O.D. (2013). Analysis of Entropy generation rate in an unsteady porous channel with Navier slip and convective cooling. Entropy, 15(6): 2081-2099. https://doi.org/10.3390/e15062081

[24] Adesanya, S.O., Dairo, O.F., Yusuf, T.A., Onanaye, A.S. Arekete, S.A. (2019). Thermodynamics Analysis for heated gravity-driven hydromagnetic Couple Stress film with viscous dissipation effects. Physica A: Statistical Mechanics and its Applications, 540: 123150. https://doi.org/10.1016/j.physa.2019.123150

[25] Dalir, N., Dehsara, M., Nourazar, S.S. (2015). Entropy analysis for magnetohydrodynamic flow and heat transfer of a Jeffrey nanofluid over a stretching sheet. Energy, 79: 351-362. https://doi.org/10.1016/j.energy.2014.11.021

[26] Opanuga, A.A., Agboola, O.O., Gbadeyan, J.A., Okagbue, H.I. (2020). Entropy generation analysis of Hall current effect on MHD micropolar fluid flow with rotation effect. SN Applied Sciences, 2: 18. https://doi.org/10.1007/s42452-019-17837

[27] Nadeem, S., Hussain, S.T., Lee, C. (2013). Flow of a Williamson fluid over a stretching sheet. Brazilian Journal of Chemical Engineering, 30(03): 619-625. https://doi.org/10.1590/S0104-66322013000300019

[28] Nadeem, S., Akbar, N.S. (2011). Numerical solutions of peristaltic flow of Williamson fluid with radially varying MHD in an Endoscope. Int. J. Numer. Meth. Fluids, 66(2): 212-220. https://doi.org/10.1002/fld.2253

[29] Nagendra, N., Amanulla, C.H., Reddy, M.S. (2017). Slip effects on MHD flow of a Williamson fluid from an isothermal sphere: A numerical study. AMSE JournalsAMSE IIETA publication-2017-Series: Modelling B, 86(3): 782-807.https://doi.org/10.18280/mmc_b.860311

[30] Ayaz, F. (2004). Application of differential transform method to differential-algebraic equations. Applied Mathematics and Computation, 152(3): 649-657. https://doi.org/10.1016/S0096-3003(03)00581-2

[31] Mohammadyari, R., Rahimi-Esbo, M., Khalili, A.A. (2014). Differential transformation method to determine magneto hydrodynamics flow of compressible fluid in a channel with porous walls. Boletim da Sociedade Paranaense de Matematica., 32(2): 249-261. https://doi.org/10.5269/bspm.v32i2.21618 